\title{
Power Allocation for the Fading Relay Channel with Limited Feedback
}

\author{
Yong Liu \\ Department of Electronic Engineering, Shanghai Jiaotong University, Shanghai, P. R. China, 200240, \\ Email: yongliu1982@sjtu.edu.cn
}

Wen Chen

Department of Electronic Engineering, Shanghai Jiaotong University, Shanghai, P. R. China, 200240, SKL for Mobile Communications, Southeast University, P. R. China, 211189, Email: wenchen@sjtu.edu.cn,

Jietao Zhang, and Zezhou Luo

Communication Technologies Department, Huawei Technologies Co., Ltd., Shenzhen, P. R. China, 518129

Email: \{jtzhang;luozezhou\}@ huawei.com

\begin{abstract}
It has been shown that channel state information (CSI) at transmitter can significantly increase the performance of a relay system. However, most of the existing designs assume perfect CSI at the transmitters. Since most practical systems can only obtain partial CSI at the transmitters, it is necessary to analyze the relay channels with limited CSI feedback. Our objective in this paper is to find the optimal power allocation strategy for relay channel under different levels of transmitter CSI, with the system outage probability constraint. We consider a Decode-and-Forward (DF) cooperative diversity model where one source node communicates with one destination node assisted by one half duplex relay. The Lloyd Algorithm is employed to quantize the CSI at receiver and construct the codebook, whose copies are also equipped on the source and the relay nodes. Each code in the codebook is a power allocation vector. Simulation results show that a few feedback bits can significantly improve the system performance.

Index Terms-Limited Feedback, Power Allocation, Decode and Forward, Lloyd Algorithm, Relay Channel.
\end{abstract}

\section{INTRODUCTION}

The Multiple-Input Multiple-Output (MIMO) techniques have achieved distinct advances in the past decade [1], since it is able to provide high capacity and throughput without increasing system bandwidth. However, many wireless devices are limited by size or hardware complexity for multiple antennas, more and more attention is paid to relay cooperation technology in recent years. Relay cooperation is an alternative technology for obtaining spatial diversity in wireless networks [2], [3], in which relays are assigned to assist source in forwarding its information to the destination, thus forming a virtual antenna array.

It is well known that resource allocation employing channel adaptive signaling can yield significant improvement in almost any performance metric of relay channels [4], [5], [6], [7]. The tremendous innovation that realized instantaneous channel adaptation practice is the use of feedback whose history may trace back to Shannon [8], [9]. However, most previous works assume perfect CSI at relay and the source. It has been shown that the outage performance with perfect CSI at the receiver and transmitter for a MIMO system can be significantly improved compared to that without CSI at transmitter [10]. The works in [11], [12] have also shown that capacity of fading channels are significantly improved with perfect CSI feedback. The authors of [13] utilize power control and adaptive signaling to increase the rate for a given BER using perfect CSI at the transmitters in single antenna systems. However, practical systems seldom have perfect CSI feedback due to various resources, such as estimation errors, feedback delay and feedback errors, etc.. It is desirable to investigate the system performance with partial CSI at transmitters.

The MIMO systems with partial CSI at transmitters have been widely investigated [14], [15], [16], [17]. The authors of [18] consider power control for Amplify-and-Forward (AF) relaying systems with quantized CSI feedback from the destination, in which the diversity analysis is also performed. However, the outage probability observed in [18] is only a loose lower bound. From a diversity-multiplexing tradeoff point of view, the authors in [19] use statistical channel knowledge to optimize the dimension allocation.

In this paper, we consider the Decode-and-Forward (DF) cooperative model with one source node communicate with one destination node assisted by a half-duplex relay, subject to slowly but randomly varying channel gains. We propose a power allocation strategy under different levels of quantized CSI feedback using Lloyd algorithm. In our feedback scheme, we consider that the destination feedbacks CSI to the source and the relay. The Lloyd Algorithm is employed to quantize the CSI at receiver and construct the codebook, whose copies are also equipped on the source and the relay nodes. Each code in the codebook is a power allocation vector. Simulation results show that a few feedback bits can significantly improve the system performance. 


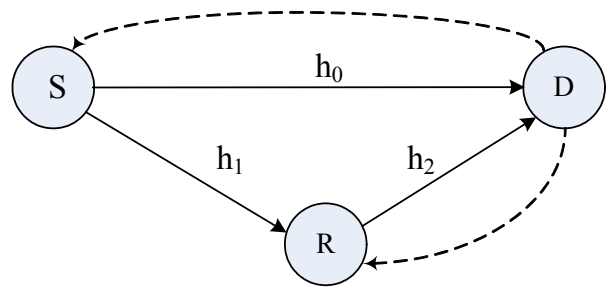

Fig. 1. Three-node relay channel with limited feedback

The rest of the paper is organized as follows. In Section II, we briefly describe the system model. In Section III, we drive the outage probability for the relay system with limited feedback. In Section IV, we present simulation results to demonstrate the analysis. Finally the conclusions are made in section $\mathrm{V}$.

\section{SYSTEM MODEL}

The relay system considered in this paper is depicted in Fig. 1, with one relay node and one source-destination pair. The relay does not produce its own data and it only assists in the communication between the source and the destination. We assume that all the nodes work in the half-duplex mode. Communication between the source and destination takes place in two time slots. In the first time slot, the source broadcasts its information, the relay and the destination receive; while in the second time slot, the relay transmits what it has received in the first time slot to the destination. Then the destination tries to decode based on all the signals received during both time slots.

The relay can carry out various kinds of information processing, among which Amplify and Forward (AF) and Decode and Forward (DF), were introduced in [20]. We use DF scheme in this paper. According to the DF protocol, the system mutual information $I$ depends on the worst channel condition, that is

$$
I=\min \left\{\log \left(1+\frac{1}{2} \rho_{s, r}\right), \log \left(1+\frac{1}{2}\left(\rho_{s, d}+\rho_{r, d}\right)\right)\right\},
$$

where $\rho_{s, d}, \rho_{r, d}, \rho_{s, r}$ denote instantaneous received SNR rates of the channels $h_{0}, h_{1}$ and $h_{2}$ respectively.

The received signal at both the source and the relay is corrupted by additive white Gaussian noise with zero mean and unit variance. The $h_{i}$ for $i=0,1,2$ denote the fading coefficient. Then the received signal at the destination and relay in the time slot 1 are respectively

$$
\begin{aligned}
& y_{1}=h_{0} * x_{1}+z_{1}, \\
& y_{r}=h_{1} * x_{1}+z_{r},
\end{aligned}
$$

where $x_{1}$ is the signal transmitted by the source at time slot 1 , $z_{1}$ and $z_{r}$ are the Gaussian noise at the destination and the relay respectively. In the time slot 2 , the received signal at the destination is

$$
y_{2}=h_{2} * x_{r}+z_{2},
$$

where $x_{r}$ is the signal transmitted by relay and $z_{2}$ is the Gaussian noise at the destination in time slot 2 . We consider block fading model, where the fading coefficients $\gamma_{i}=\left|h_{i}\right|^{2}$ keep constant over one block and are independently change from one block to the next one. Outage probability is a practical analysis measurement for the block fading environment, which denotes the probability of event that the instantaneous mutual information $I$ is smaller than the data rate $R$, that is

$$
P_{\text {out }}=\operatorname{Prob}\left(I\left(P_{s}, P_{r}, \bar{\gamma}\right)<R\right),
$$

where $P_{s}$ denotes transmit power of the source, $P_{r}$ is power of the relay, and $\bar{\gamma}=\left(\gamma_{0}, \gamma_{1}, \gamma_{2}\right)$. The system is said to achieve a diversity order of $d$ if its outage probability satisfies

$$
P(r \log (S N R)) \doteq S N R^{-d},
$$

where $\doteq$ denotes the equivalence in the sense of $S N R \rightarrow \infty$.

\section{Optimal Power Allocation with Limited FEEDBACK}

In this section, we present a optimal power allocation algorithm based on limited CSI feedback. First, we outline the general procedure. Then we will analyze the proposed algorithm elaborately.

\section{A. General Procedure}

We consider $\gamma_{i}$ for $i=0,1,2$ follow the exponential distribution with mean $\lambda_{i}$, where $\lambda_{i}$ denotes the channel's path-loss. $\lambda_{i}$ depends on the link length $d_{i}$ and the path-loss exponent $\alpha$, i.e., $\lambda_{i}=d_{i}^{-\alpha}$.

Suppose that the destination has a perfect CSI $\bar{\gamma}$. Then it would be quantized at first. If the destination node carry out $K$ bits feedback, the CSI space will be quantized into $H$ (= $2^{K}$ ) regions. We will adopt Lloyd algorithm [21] to quantize the CSI space. The destination, upon the current $\bar{\gamma}$, selects a power vector $P_{q}=\left(P_{s, q}, P_{r, q}\right)$ from the designed codebook $\mathbb{C}$ of size $H$. Then it broadcasts the index $q$ to both the source and relay. Upon the received index $q$, the source transmits with power $P_{s, q}$ and the relay transmits with power $P_{r, q}$. The elements of the codebook are designed to maintain the power constraints of both the source and relay, i.e., elements of each power allocation vector should satisfy the long-term power constraint [5]:

$$
\left(P_{s}, P_{r}\right)=\left(\sum_{q=1}^{H} P_{s, q} \int_{\mathfrak{R}_{\mathfrak{q}}} f(\bar{\lambda}) d \bar{\lambda}, \sum_{q=1}^{H} P_{r, q} \int_{\mathfrak{R}_{\mathfrak{q}}} f(\bar{\lambda}) d \bar{\lambda}\right),
$$

where $\bar{\lambda}=\left(\lambda_{0}, \lambda_{1}, \lambda_{2}\right)$, and $f(\bar{\lambda})$ denotes the joint probability distribution of $\bar{\lambda}$. Apparently, the case that both the source and relay have individual average power constraints is considered here.

Then the question lies in the design of the so called power control mapping $\mathbf{F}: \mathfrak{R} \rightarrow \mathbb{C}$, where $\mathfrak{R}$ is the CSI space, with the average power constraint $\mathbb{E}_{\bar{\gamma}}(\mathbf{F}(\bar{\gamma})) \leq\left(P_{s}, P_{r}\right)$, which hold on a per-element basis. Thereby, the essence of our algorithm is to realize the objective of finding an optimal $\mathbf{F}(\bar{\gamma})$ in order to minimize system outage probability while meeting the power constraint. 


\section{B. Lloyd Algorithm Based Codebook Design}

As mentioned above, codebook design is a crucial point in the proposed algorithm. In this section, we will employ Lloyd algorithm to construct the codebook.

The key points of Lloyd algorithm are centroid rule and nearest neighborhood rule [21], which are iteratively used in our codebook optimization. Actually the centroid rule aims to select the optimal codeword to minimizes the system outage probability in a region, while the latter is designed to determine the region in which the vectors in this region are closest to the codeword of this region. We have to produce the training vectors $\left(T_{i} \mid i=0,1, \ldots, M\right) \subset \mathfrak{R}$ with $M \gg H$. Then the algorithm can be expressed as follows.

1) Randomly select the initial codebook $\left\{C_{q} \mid q=\right.$ $1,2, \ldots, H\}$ from the training vectors $\left(T_{i} \mid i=\right.$ $0,1, \ldots, M)$;

2) Cluster the training vectors into $H$ groups $R_{q}=$ $\left\{T_{i} \mid d\left(T_{i}, C_{q}\right)<d\left(T_{i}, C_{p}\right), p \neq q\right\}$ for $q=1,2, \ldots, H$, where $d\left(T_{i}, C_{q}\right)$ represents the Euclidean distance between $T_{i}$ and $C_{q}$.

3) We defined the minimum distance of the codebook as

$$
d_{\text {min }}=\min _{C_{q}}\left(\frac{1}{N_{R_{q}}} \sum_{T_{v} \in R_{q}} d\left(T_{v}, C_{q}\right)\right),
$$

where $N_{R_{q}}$ is the number of the training vectors in $R_{q}$. If $d_{\text {min }}$ decreases, go to the next step; Otherwise stop iteration.

4) Given a region $R_{q}$, design the new codeword by

$$
C_{q}^{*}=\arg \min _{C_{q}}\left(\frac{1}{N_{R_{q}}} \sum_{T_{v} \in R_{q}} d\left(T_{v}, C\right)\right),
$$

Then go to step 2. Using the centroid rule step 4 and the nearest neighborhood rule step 2 iteratively, the system outage probability will decreases monotonically.

\section{Outage Performance Analysis}

The performance of the transmission scheme with diversity employing DF relaying is estimated in terms of outage probability. It has been shown that the average mutual information in a DF diversity transmission scheme depends on the worst channel condition. Then the outage probability is given by

$$
P_{\text {out }}=\operatorname{Prob}\left\{\min \left(\rho_{0}, \rho_{1}, \rho_{2}\right) \leq \rho_{t}\right\},
$$

where $\rho_{t}$ denotes a given $S N R$ threshold, and $\rho_{i}$ for $i=0,1,2$ denote each channel's SNR. Let $G_{t}$ and $G_{d}$ are the gains of transmitter and the destination respectively. Let

$$
\overline{\rho_{s}}=\frac{G_{t} G_{d} \kappa^{2}}{(4 \pi)^{2} d^{\alpha}} P_{s}, \quad \overline{\rho_{r}}=\frac{G_{t} G_{d} \kappa^{2}}{(4 \pi)^{2} d^{\alpha}} P_{r},
$$

where $\kappa$ is a constant and $\rho$ is a SNR variable. Then for our scheme, the outage probability

$$
P_{\text {out }}=1-\int_{\rho_{t}}^{\infty} \frac{1}{\overline{\rho_{s}}} e^{-\left(\frac{\rho}{\rho_{s}}\right)} d \rho \int_{\rho_{t}}^{\infty} \frac{1}{\overline{\rho_{r}}} e^{-\left(\frac{\rho}{\rho_{r}}\right)} d \rho
$$

It is well known that

$$
\begin{aligned}
& \int_{\rho_{t}}^{\infty} \frac{1}{\overline{\rho_{s}}} e^{-\left(\frac{\rho}{\rho_{s}}\right)} \mathrm{d} \rho \int_{\rho_{t}}^{\infty} \frac{1}{\overline{\rho_{r}}} e^{-\left(\frac{\rho}{\rho_{r}}\right)} \mathrm{d} \rho \\
& =\int_{\rho_{t}}^{\infty} \frac{1}{\overline{\rho_{s}} \cdot \overline{\rho_{r}}} e^{-\left(\frac{\rho}{\rho_{s}}+\frac{\rho}{\rho_{r}}\right)} \mathrm{d} \rho \\
& =e^{-\rho_{t}\left(\frac{1}{\overline{\rho_{s}}}+\frac{1}{\rho_{r}}\right)},
\end{aligned}
$$

that is

$$
P_{\text {out }}=1-e^{-\rho_{t}\left(\frac{1}{\rho_{s}}+\frac{1}{\rho_{r}}\right)} .
$$

Let

$$
G_{s}=\frac{G_{t} G_{d} \kappa^{2}}{(4 \pi)^{2} d^{\alpha}}, \quad G_{r}=\frac{G_{t} G_{d} \kappa^{2}}{(4 \pi)^{2} d^{\alpha}} .
$$

Then the outage probability can be transformed as

$$
P_{\text {out }}=1-e^{-\rho_{t}\left(\frac{1}{G_{s} P_{s}}+\frac{1}{G_{r} P_{r}}\right)} .
$$

So the optimization issue becomes

$$
\begin{aligned}
& \min \quad P_{s}+P_{r} \\
& \text { s.t. } \quad P_{\text {out }} \leq P_{c},
\end{aligned}
$$

where $P_{c}$ is the outage probability constraint. The solution can be found as follows.

$$
\begin{array}{ll}
\min & P_{s}+P_{r} \\
\text { s.t. } & \frac{1}{G_{s} P_{s}}+\frac{1}{G_{r} P_{r}} \leq-\frac{\ln \left(1-P_{c}\right)}{\rho_{t}} .
\end{array}
$$

Then we use Lagrange multiplier to find the optimal power allocation vector. Let

$$
f\left(P_{s}, P_{r}\right)=P_{s}+P_{r}+\mu\left(\frac{1}{G_{s} P_{s}}+\frac{1}{G_{r} P_{r}}+\frac{\ln \left(1-P_{c}\right)}{\rho_{t}}\right) .
$$

Then

$$
\frac{\partial f}{\partial P_{s}}=1-\frac{\mu}{G_{s} P_{s}^{2}}=0, \quad \frac{\partial f}{\partial P_{r}}=1-\frac{\mu}{G_{r} P_{r}^{2}}=0 .
$$

Then we obtain

$$
P_{s}=\sqrt{\mu / G_{s}}, \quad P_{r}=\sqrt{\mu / G_{r}}
$$

Therefore, we have

$$
\frac{1}{\sqrt{\mu G_{s}}}+\frac{1}{\sqrt{\mu G_{r}}}=-\frac{\ln \left(1-P_{c}\right)}{\rho_{t}} .
$$

This implies

$$
\sqrt{\mu}=-\left(\frac{1}{\sqrt{G_{s}}}+\frac{1}{\sqrt{G_{r}}}\right) \frac{\rho_{t}}{\ln \left(1-P_{c}\right)} .
$$

Then the optimal solution of $P_{s}$ and $P_{r}$ are respectively

$$
\begin{aligned}
& P_{s}^{*}=\frac{\rho_{t}\left(\sqrt{G_{s}}+\sqrt{G_{r}}\right)}{G_{s} \sqrt{G_{r}} \ln \left(1-P_{c}\right)}, \\
& P_{r}^{*}=\frac{\rho_{t}\left(\sqrt{G_{s}}+\sqrt{G_{r}}\right)}{G_{r} \sqrt{G_{s}} \ln \left(1-P_{c}\right)} .
\end{aligned}
$$

By now, we have found a pair of optimal power vector $\left(P_{s}^{*}, P_{r}^{*}\right)$ which realized the minimization of the total transmission power. Our analysis can be extended to transmission schemes with multiple relays, in which, the only difference lies in the expression of $P_{\text {out }}$. 


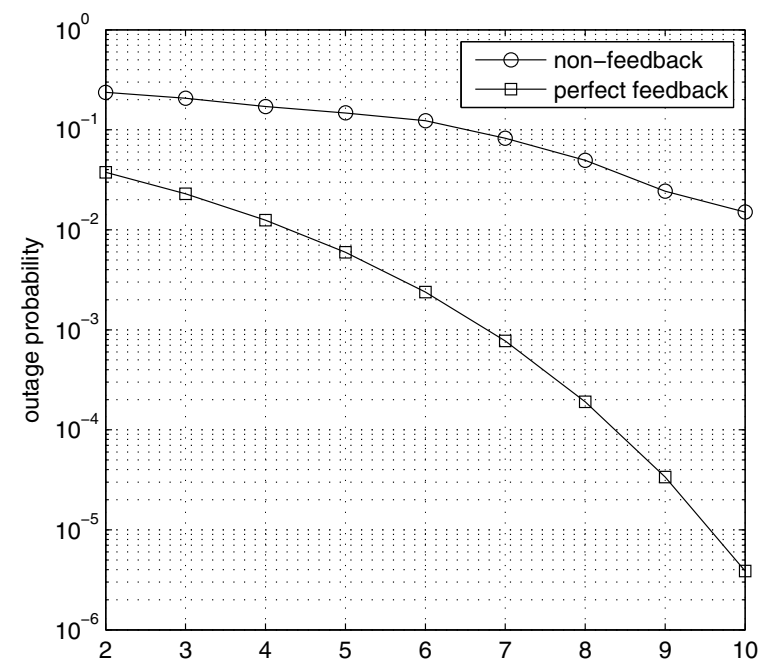

Fig. 2. Outage performance versus SNR for the DF scheme, the upper curve denotes Constant power transmission, and the other curve indicates perfect power allocation scheme

\section{NUMERICAL RESULTS}

In this section, we present some numerical results to demonstrate the performance of the power allocation algorithm. We adopt Monte Carlo simulation for the DF relay system. We assume that the magnitudes of channel fading coefficients $h_{i}, i \in\{0,1,2\}$ follow a Rayleigh distribution and are independent of each other. The received signals at both the source and relay are corrupted by additive white Gaussian noise with unit variance. We assume the path-loss exponent $\alpha=3.5$. Constant power transmission is compared to the proposed power control strategy with perfect CSI based power allocation algorithm in Fig. 2. It can be clearly found that the proposed power allocation policy achieves tremendous gains over the fading channel. In the figure, the upper curve denotes the system outage probability of constant power transmission strategy, whose source and relay are given equal average power. The lower curve is the lower bound of outage probability, which can be achieved via adopting perfect CSI in the threenode. If we compare the power consumed by the two schemes, the superiority of perfect CSI based optimal power allocation scheme is apparent. At a given outage probability of $10^{-2}$, more than $8 d B$ savings in power over the constant power transmission is achieved through optimal power allocation. This simulation motivates us to investigate the DF cooperative system with partial CSI feedback.

We now validate the effect of the proposed scheme with limited feedback, and the result is shown in Fig. 3. We compare the optimal power control protocol with different levels of feedback bits. We can find that only a few feedback bits can significantly improve the system performance. Further increasing in the feedback bits will continue improve the performance, but the improvement becomes limited. In Fig. 3, with 1 bit of feedback at an outage probability of $10^{-2}$, there

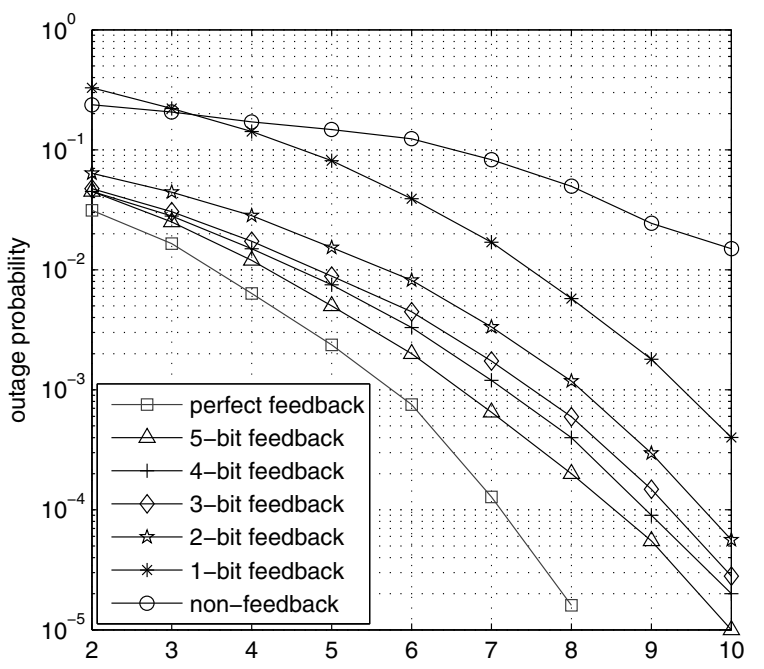

Fig. 3. Effect of different feedback bits on outage performance versus SNR, using the DF protocol, the lowest curve indicates perfect power allocation scheme. Additionally, the outage performance of transmission system using constant power is presented

is only a $-4 \mathrm{~dB}$ gap to the bench mark. Besides that, the performance gap between $N=3, N=4$ and $N=5$ is decreasing. The simulation implies that the feedback bits as well as the size of codebook in our model is not necessarily to be very large.

As mentioned above, we employed Lloyd algorithm to design power allocation codebook. In Fig. 4, we show convergence performance of the algorithm. We adopt the outage probability difference of each iteration as measurement. Fig. 4 presents a realization of the achieved outage probability difference during each iterations. We can find that the system outage probability difference decreases very fast, which means the iterative algorithm quickly converges. It implies that a few times of iteration is enough for the Lloyd algorithm.

\section{CONClusion}

In this paper, we have proposed a power allocation strategy employing different degrees of CSI at the source and relay in the fading DF relay channel. Provided partial channel state information is available at the source and relay, significant power savings will be achieved via optimal power control over constant power transmission, and tremendous gain is obtained in system outage probability. Moreover, we proved that only a few bits of feedback are enough to offer significant improvement for the system by simulation.

\section{ACKNOWLEDGEMENT}

This work is supported by NSF China \#60972031, by SEU SKL project \#W200907, by Huawei Funding \#YJCB2009024WL and \#YJCB2008048WL, and by National 973 project \#2009CB824900. 


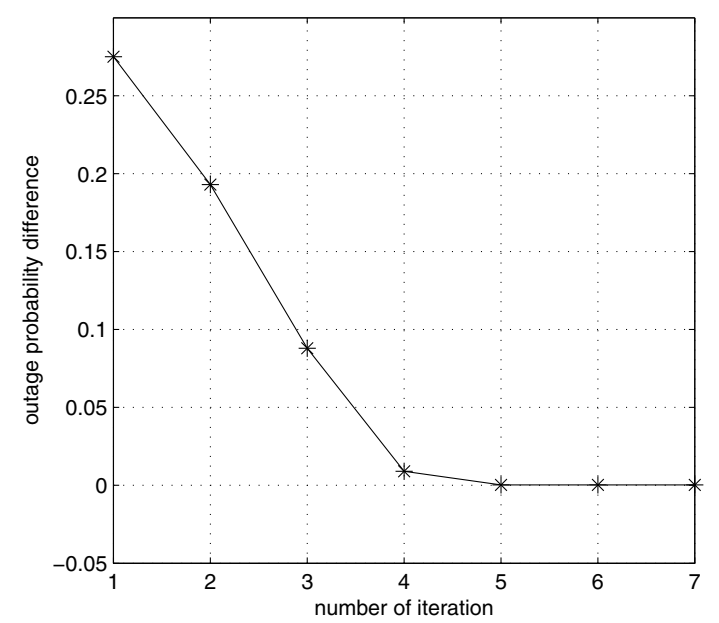

Fig. 4. Convergence performance of the proposed algorithm

\section{REFERENCES}

[1] A. J. Paulraj, D. A. Gore, R. U. Nabar, et al., " An overview of MIMO communications C A Key to Gigabit Wireless," Proceeding of the IEEE, Vol. 92, no. 2, pp. 198-218, Feb. 2004.

[2] A. Sendonaris, E. Erkip, and B. Aazhang, " User cooperation diversityPart I: System description," IEEE Trans. Commun., vol. 51, no. 11, pp. 1927-1938, Nov. 2003.

[3] A. Sendonaris, E. Erkip, and B. Aazhang, " User cooperation diversityPart II: Implementation aspects and performance analysis," IEEE Trans. Commun., vol. 51, no. 11, pp. 1939-1948, Nov. 2003.

[4] M. O. Hasna and M. S. Alouini," Optimal power allocation for relayed transmissions over Rayleigh-fading channels," IEEE Trans. Wireless Commun., vol. 6, pp. 1999-2004, Nov. 2004.

[5] G. Caire, G. Taricco, and E. Biglieri, " Optimum power control over fading channels," IEEE Trans. Inf. Theory, vol. 45, pp. 1468-1489, Jul. 1999.

[6] T. T. Kim and M. Skoglund, " Diversity-multiplexing tradeoff in MIMO channels with partial CSIT," IEEE Trans. Inf. Theory, vol. 53, pp. 27432759, Aug. 2007.

[7] I. Maric and R. D. Yates, " Bandwidth and power allocation for cooperative strategies in Gaussian relay networks," in Proc. Asilomar Conf. Signals, Syst., Comput., Pacific Grove, CA, Nov. 2004, pp. $1907-$ 1911.

[8] C. E. Shannon," The zero error capacity of a noisy channel," IRE Trans. Inform. Theory, vol. 2, no. 3, pp. 8-19, Sept. 1956.

[9] C. E. Shannon, "Channels with side information at the transmitter," IBM Journal Research and Dev., vol. 2, pp. 289-293, 1958.

[10] S. Bhashyam, A. Sabharwal, and B. Aazhang, "Feedback gain in multiple antenna systems," IEEE Trans. Commun., vol. 50, no. 5, pp. 795-798, May 2002.

[11] T. Yoo, and A. Goldsmith, "Capacity and Power Allocation for Fading MIMO Channels With Channel Estimation Error,' IRE Trans. Inform. Theory, vol. 52, no. 5, pp. 2203-2214, May 2006.

[12] S. Tatikonda, and S. Mitter, "The Capacity of Channels With Feedback," IRE Trans. Inform. Theory, vol. 55, no. 1, pp. 323-349, Jan. 2009.

[13] N. Prasad, and M. K. Varanasi, "Analysis of decision feedback detection for MIMO Rayleigh-fading channels and the optimization of power and rate allocations," IRE Trans. Inform. Theory, vol. 50, no. 6, pp. 10091025, Jun. 2004.

[14] D. J. Love and R. W. Heath Jr., Limited feedback precoding for spatial multiplexing systems, IEEE Trans. Information Theory, vol. 51, no. 8, Aug., 2005.

[15] S. L. Zhou, BER criterion and codebook construction for finite-rate precoded spatial multiplexing with linear receiver, IEEE Trans. signal processing, vol. 50, no. 10, pp. Oct. 2006.
[16] P. Xia, Design and analysis of transmit-beamforming based on limitedrate feedback, IEEE Trans. Signal Processing. vol. 54, no. 5, pp. 18531863, May 2006.

[17] V. Lau, Y. Liu, and T.-A. Chen, On the design of MIMO block-fading channels with feedback-link capacity constraint, IEEE Trans. Commun., vol. 52, no. 1, pp. 62-70, Jan. 2004

[18] N. Ahmed, M. A. Khojastepour, A. Sabharwal, and B. Aazhang," Outage minimization with limited feedback for the fading relay channel," IEEE Trans. Commun., vol. 54, pp. 659-669, Apr. 2006.

[19] P. Elia, K. Vinodh, M. Anand, and P. V. Kumar, " D-MG tradeoff and optimal codes for a class of AF and DF cooperative communication protocols," IEEE Pro. Inf. Theory, pp. 681-685, Jun. 2007.

[20] G. Kramer, M. Gastpar, and P. Gupta, " Cooperative Strategies and Capacity Theorems for Relay Networks," IEEE Trans. Inf. Theory, VOL. 51, NO. 9, pp. 3037-3062, Sep. 2005.

[21] A. Gersho and R. M. Gray, " Vector Quantization and Signal Compression," Kluwer Academic Publishers, Boston, 1992. 